\title{
Augmented Reality: Can it Enrich the Practice of Anesthesiology?
}

Augmented reality is a term coined in 1992 by Tom Caudell, used to define a direct or indirect view of the physical realworld combined with virtual elements, creating a live mixed reality. Augmented reality devices typically consist of a combined headset, GPS, and display system to show the user virtual information that is actually added to reality. Augmented reality offers endless possibilities for interaction, allowing its application in many areas.

In medicine, the most evident example would be that of Brigham and Women's Hospital in Boston, Massachusetts ${ }^{1}$, which has a surgical planning laboratory in the neurosurgery department, where around 200 procedures have been completed using augmented reality technology.

The current application of augmented reality in Anesthesiology is represented by well-known instances of virtual reality, such as that provided by head-mounted displays (HMD). These devices overlap computer-generated images with a real field of vision ${ }^{2}$. HDMs allow patient's vital signs to be overlaid onto anesthesiologists' visual field regardless of mon- itor location. This would theoretically help anesthesiologists to monitor vital signs and detect "intraoperative incidents" faster than in the traditional system ${ }^{3}$. Anesthesiology studies using augmented reality are sparse, from a very small sample of anesthesiologists and under simulated conditions, yet the results are promising ${ }^{3,4}$. Although its use in Anesthesiology currently presents limitations, augmented reality represents a starting point for future clinical trials of HMD device use by anesthesiologists for intraoperative monitoring, providing all the patient information in a virtual screen with its global and focal vision.

Ana Sofía Del Castillo Anesthesiologist, Hospital del Niño, Panama City, Panama

Norma S. Sardi Pathologist, Hospital Santo Tomas, Panama City, Panama 


\section{REFERENCES}

1. Disponível em: http://groups.csail.mit.edu/vision/medical-vision/links. html.

2. Liu D, Jenkins SA, Sanderson PM - Patient Monitoring with head. mounted displays. Curr Opin Anaesthesiol, 2009;22:796-803.

3. Sanderson PM, Watson MO, Russel WJ et al. - Advanced auditory displays and head-mounted displays: advantages and disadvantages for monitoring by the distracted anesthesiologist. Anesth Analg, 2008;106:1787-1797.

4. Liu D, Jenkins SA, Sanderson PM, Fabian P, Russell WJ - Monitoring with head-mounted displays in general anesthesia: a clinical evaluation in the operating room. Anesth Analg, 2010;110:1032-1038. 